\title{
ERZÄHLEN MIT BIBLISCH-LITURGISCHEN TEXTEN ${ }^{1}$
}

\begin{abstract}
Summary: The author's thesis is that from the beginning of the establishment of the so-called FrankoRoman Liturgy (thus at the latest from the Carolingian era) the texts of the Bible were primarily mediated to the literate population through the liturgical and liturgical-musical culture. Thus the quotation of Biblical texts was greatly influenced by their liturgical use, and the knowledge connected to them as a result of it. Texts written for a wider audience were based on passages common in, or only found in liturgical use. Such are the pericopes (designated passages of the Bible) of larger Holidays and more common events, and the liturgical elements sung connected to these Holidays, or other important dates. This background knowledge influenced not only works of a religious nature, but also chiefly secular texts, among these historiographic writings. The paper analyses a short excerpt from a Hungarian chronicle (Chronici Hungarici compositio saeculi $X I V$, c. 164), proving that it is a cento of commonly used, and thus well-known liturgical texts from the Bible.
\end{abstract}

Key words: Bible, Latin liturgy, Centonisation, Chronici Hungarici compositio saeculi XIV

Vor einigen Jahren habe ich an dem vielleicht wichtigsten Text der Latinität der Árpád-Zeit, an den Admonitiones, die mit dem Namen des ersten Königs von Ungarn, des Heiligen Stephanus, verbunden sind, beobachtet, dass drei früher für ungenaue biblische Zitate gehaltene Stellen eigentlich die ersten Teile von Responsorien sind. Alle drei Sätze stammen aus demselben Matutinum des Monats August, der Reihe Historia Sapientiae (HSap). Das „,biblische“ Zitat, das am Anfang des ganzen Werkes steht, ist der Anfang des Responsoriums Audi fili mi disciplinam. An einer späteren Textstelle werden zwei Sätze, die auch im Stundengebet direkt nacheinander gesungen

\footnotetext{
${ }^{1}$ Eine frühere Version des Artikels: DÉRI, B.: Krónika és liturgia [Chronik und Liturgie]. Magyar Egyházzene 20 (2012) 37-42. Eine spätere Version wurde als Vortrag im Rahmen des Kolloquiums „Narratologie und Erzähltechnik“ (in Kooperation des Instituts für Altertumswissenschaft, ELTE, des Instituts für Klassische Philologie, Mittel- und Neulatein der Universität Wien und der Universität Salzburg), 7-8. November 2013, in Budapest vorgestellt.
} 
werden, Domine Pater et Deus und Emitte Domine sapientiam, ausdrücklich nebeneinander zitiert. ${ }^{2}$

Sofort zeigte sich, dass ihre textlichen Abweichungen von den maßgebenden wissenschaftlichen lateinischen Bibelausgaben oder sogar von dem mittelalterlichen Textzustand der Bibel nicht auf das leider allzu oft angenommene ungenaue Gedächtnis des Verfassers oder auf die absichtliche literarische Transformation, auf die Lust am Paraphrasieren zurückzuführen sind. Dies sind genaue Zitate, aber nicht direkt aus der Bibel. Es handelt sich um liturgische Texte, Centos, die zum Teil auf Textvarianten basieren, die älter sind als die Vulgata. Diese Texte sind in der Tat durch ein Verfahren des Paraphrasierens und Centonisierens entstanden, aber mehrere Jahrhunderte vor den Admonitiones.

Meine früheren Vermutungen in dieser Frage ${ }^{3}$ erhielten eine feste Kontur. $\mathrm{Zu}$ erst habe ich die Admonitiones analysiert, dann die (ganze) lateinische Prosaliteratur (historische Literatur, Legenden) und theologische Literatur des frühmittelalterlichen Ungarns (d.h. vor dem Renaissance-,,Humanismus“), um nach der biblischen und biblisch-liturgischen Wirkung zu suchen. (Absichtlich rede ich nicht von „Zitaten“.) Ich habe mir auch einen Überblick über die dichterischen Texte verschafft, aber die Homiletik noch nicht unter die Lupe genommen. (Deren größter Teil ist Produkt des scholastischen Predigtstils.) Ein Teil der Ergebnisse sind schon veröffentlicht worden, ${ }^{4}$ ihr Großteil liegt aber noch in Manuskriptform vor.

Diese Forschung soll zur genaueren Beschreibung des Verhältnisses der Texte zur Lateinischen Bibel als Hypotext beitragen, das viel komplizierter ist, als bislang angenommen. Auch der philologische Ertrag, d.h. die Identifizierung der einzelnen Textstellen, ist vielleicht nicht ohne Bedeutung.

Viel wesentlicher ist aber, dass 1) die außergewöhnliche Bedeutung der liturgischen und liturgisch-musikalischen Kultur sich auch im Falle der Entstehung nicht liturgischer Texte gezeigt hat, und dass 2) man mit dem literaturwissenschaftlichen Begriff der Vermittlungsmedien wie mit rezeptionsästhetischen/-theoretischen Überlegungen das Publikum (die „Zielgruppe“) der Werke beschreiben kann.

Ich formuliere thesenartig. Von der Fixierung der Grundlinien der sogenannten fränkisch-römischen Liturgie europaweit (also spätestens seit der Karolingerzeit) bis zum Ende des Mittelalters (dieses „Ende“ hat sich in einigen Kontexten und auf einigen Gebieten weit über das im historischen Sinne übliche Mittelalter hinaus ausgedehnt) ordnen sich die literarischen Texte, die textuell eine biblische Wirkung aufzei-

\footnotetext{
${ }^{2}$ Mit liturgiewissenschaftlichen Abkürzungen: responsorium Domine Pater et Deus (CAO-6503, HSap N R, cf. Eccli 23,4-6), responsorium Emitte Domine sapientiam (CAO-6657, HSap N R, cf. Sap 9,10), responsorium Audi fili mi disciplinam (CAO-6140, HSap N R, Prv 1,8 et 4,10).

${ }^{3}$ Ein erster Versuch: DÉRI, B.: „... Et pigmenta multa habebant.“ (Megjegyzések P. mester forrásainak néhány kérdéséhez) [Bemerkungen zu einigen Problemen der Quellen des Magister P.]. Magyar Könyvszemle 105 (1989) 223-236, hier 234, Anm. 45.

${ }^{4}$ DÉRI, B.: Három liturgikus tétel Szent István Intelmeiben [Drei liturgische Sätze in den Admonitiones des Heiligen Stephanus]. Irodalomtörténeti Közlemények 114 (2010) 3-16. DÉRI, B.: Egy nagyböjti responzórium szövege legendáinkban? [Der Text eines Responsoriums der Fastenzeit in unseren Legenden?]. Magyar Egyházzene 18 (2010/2011) 363-366. Über Anonymus und Simon Kézai s. Anm. 5 und 6.
} 
gen - v.a. die lateinischen, aber mehr oder minder auch die volkssprachlichen -, zwischen zwei Extremen. Die Texte, die für das breiteste Publikum vorgesehen waren, wurden vor allem oder sogar ausschließlich den durch die liturgische Ordnung hervorgehobenen, sehr gut bekannten Bibeltexten entnommen. Solche sind die Perikopen (für das Vorlesen/Rezitieren vorgesehene Bibelausschnitte) der wichtigen Feiertage oder sich oft wiederholender Anlässe - wie die gesungenen liturgischen Sätze, die sich oft wiederhol(t)en, oder sich an wichtigen Feiertagen anschlossen bzw. auf andere Art hervorgehoben waren. Deren Bekanntheit konnte einen so hohen Grad erreichen, dass man sie ohne besonderen Hinweis oder nur anhand eines ungefähren Hinweises und sogar in einer starken Paraphrase identifizieren konnte. Die Länge der Zitate übersteigt selten die Länge eines gesungenen liturgischen Satzes.

Das andere Extrem: Die Autoren von Schriften, die für ein theologisch gebildetes, die Bibel gut kennendes elitäres Publikum vorgesehen waren, konnten frei und manchmal recht in großer Länge sogar Stellen zitieren, die im Großteil des Mittelalters nur theoretisch oder gar nicht zum liturgischen Textkorpus gehörten. (Diese wurden in der genannten Epoche im Prinzip gar nicht in den liturgischen Büchern abgeschrieben, obwohl man auch sie hätte zu Ende lesen „müssen“: Das ist der Fall bei den Bibellesungen des Matutinums.)

Extreme Beispiele für Ersteres in der mittelalterlichen lateinischen Literatur Ungarns sind die Gesta (ca. 1282-1285) von Simon Kézai (Simon von Kéza) ${ }^{5}$ und im Wesentlichen die Gesta Hungarorum (um 1200) des sogenannten Anonymus (Magister P.), ${ }^{6}$ aber großenteils gehören auch die Admonitiones (um 1022) des Heiligen Stephanus hierzu. Dem anderen Extrem nähert sich die Deliberatio (vor 1046) Gerhards des Heiligen (Gerhard von Csanád), ${ }^{7}$ diese liturgische Textbetrachtung (Kontemplation), die jedenfalls reichlich auch allgemein bekannte liturgische Texte zitiert. ${ }^{8}$ Der Adressat und erhoffte Rezipient des Werkes, sei er fiktiv oder nicht, ist eine theologisch gebildete Person.

Für die Bibelverwendung der mittelalterlichen Autoren lohnt es sich, drei Hauptkategorien auseinander zu halten.

1) Direktes Zitieren aus der Bibel, wenn das Zitat nicht spezifisch oder nicht in erster Linie Teil des (auch) durch die liturgischen Bücher vermittelten Textkorpus ist;

\footnotetext{
${ }^{5}$ DÉRI, B.:A Kézai-krónika bibliai-liturgikus idézetei [Die biblisch-liturgischen Zitate der Chronik von Simon Kézai]. Magyar Egyházzene 18 (2010/2011) 59-62.

${ }^{6}$ DÉRI, B.: P. magister gestájának bibliai-liturgikus idézetei I. [Die biblisch-liturgischen Zitate der Gesta von Magister P. Teil I]. Magyar Egyházzene 19 (2011/2012) 33-38; Teil II: ebda. 19 (2011/2012) 241-250; Teil III: ebda. 19 (2011/2012) 343-352.

${ }^{7}$ Gerardi Moresenae aecclesiae seu Csanadiensis episcopi Deliberatio supra hymnum trium puerorum. Edidit Gabriel SiLAGI. Brepols, Turnhout 1978 [Corpus Christianorum Continuatio Mediaevalis XLIX].

${ }^{8}$ Diese Charakterisierung des Werks stellt die Zusammenfassung der Ergebnisse meines bevorstehenden Buches über die Zitierweise der Deliberatio dar.

${ }^{9}$ DÉRI: P. magister ( Anm. 6) 36-38. Zusammenfassend im Teil II des Artikels (n. 6) 241.
} 
2.a) Fälle der kirchlichen und v.a. liturgischen Vermittlung, und zwar Bibelzitate in (kirchlichen) Prosatexten: in Bibel-Kommentaren und v.a. in als Matutinum-Lektionen dienenden Homilien; des Weiteren in Legenden (als liturgischen Lesungen);

2.b) der Text der Bibel als Text eines gesungenen (nicht rezitierten) Satzes, oder

2.c) Perikope oder Kapitulum einer Hora, eines Festes oder anderen liturgischen Anlasses;

2.d) die liturgischen Bücher als Lesebücher, als das vollständige Textmaterial der Zusammenfassung der klerikalen Bildung, oder

3) jeder andere Text aus der intertextuellen Textur der christlichen Bildung: aus der fromm-erbaulichen spirituellen Literatur (hierzu gehören auch die Legenden mit größerem Umfang, die man nicht auszugsweise gelesen hat) und aus den verschiedensten Schriften: von den historischen Werken bis zu Sentenzen und Urkundenformeln.

Man muss aber nicht nur diese schon an sich komplizierte Situation in Betracht ziehen, sondern auch, dass ein Werk in seinem jeweiligen Zustand zeitlich ziemlich heterogene Schichten enthält. Extrem ist in dieser Hinsicht das eminent wichtige Werk der ungarischen mittelalterlichen historischen Literatur, die eigentlich aus verschiedenen Varianten „zusammengefügte“ sogenannte Chronikkomposition aus dem 14. Jahrhundert. ${ }^{10}$ Deren beide Hauptzweige sind die sogenannte Budaer und die Wiener Chronik (oder Bilder-Chronik).

Die Einleitung der Chronikkomposition ${ }^{11}$ in der Wiener Chronik zeigt charakteristische „gelehrte“, prähumanistische Zitierverfahren: Vor den nicht unbedingt allgemein bekannten, für die Liturgie nicht typischen Zitaten gibt sie auch die Kapitelnummer der biblischen Bücher an. Gleichzeitig finden wir im Körper des sehr komplex geschichteten Textes später immer wieder Teile, die auf der Liturgiekenntnis der Personen mit klerikaler Bildung basieren (und dazu gehört jeder gebildete mittelalterliche Mensch, auch die Schulkinder!).

Als Beweis für Letzteres habe ich eine kurze und typische Textstelle ausgewählt, das Kapitel 164 über die Krönung des Königs Géza II. (Auf die genaue Datierung, auf geschichtskritische und philologische Fragen wird hier nicht eingegangen.) Die Budaer Chronik beschreibt die Krönung Gézas II. im Jahr 1141 sehr wortkarg:

${ }^{10}$ Chronici Hungarici compositio saeculi XIV. Praefatus est, textum recensuit, annotationibus instruxit A. DOMANOVSZKY, in Scriptores rerum Hungaricarum tempore ducum regumque stirpis Arpadianae gestarum (im Folgenden SRH). Edendo operi praefuit E. SZENTPÉTERY. Vol. I. Budapestini 1937, 217-505.

${ }^{11}$ SRH I 239-243.

Acta Ant. Hung. 54, 2014 
Regnavit autem post eum Geysa filius eius, qui coronatus est IIII-o (sic) Kalendas Martii, in dominica Invocavit me, transactis duobus diebus post mortem patris. (SRH I 453) ${ }^{12}$

Nach ihm herrschte Géza, sein Sohn, der im Jahr 1141 am 16. Februar gekrönt wurde, am Sonntag „Invocavit me“, zwei Tage nach dem Tod seines Vaters.

Diese auch in mittelalterlichen Urkunden häufige Zeitangabe zeigt klar: Ein so kurzer lateinischer biblischer Text wie das „Invocavit me “ galt für den damaligen Menschen nicht als Zitat von Ps 90,15, sondern als Incipit des Introitus der Messe des ersten Sonntags der Fastenzeit (Invocavit me). Für einen Christen des byzantinischen Ritus deutet z.B. „der Sonntag vom Pharisäer und Zöllner" noch heute eindeutig auf das Evangelium des ersten Sonntags der Zeit vor der Fastenzeit hin. Unser lateinisches christliches Zeitgefühl, das nach dem Zweiten Vatikanischen Konzil (mit Hinweis auf dieses, aber nicht nach dessen Vorschriften) zerstört wurde, hat diesen Ansatzpunkt zum großen Teil verloren, selbst wenn die Introitus theoretisch geblieben sind. Die Mediävistik kann darauf nicht verzichten.

Die Beschreibung der Wiener Chronik ist ausführlicher, und betreffs der Zeitangabe weicht von der Budaer Chronik ab (SRH I 453):

Respexit autem Dominus Hungariam, et dedit propugnatorem gradientem in multitudine fortitudinis suae. Dedit enim Dominus regnum Geythae, puero suo. Qui in die Sanctae Caeciliae virginis coronatus est. Cuius dexteram apprehendit, et confortavit subiecitque ei gentes multas, et dorsa regum vertit in fugam a facie gladii eius.

Es sah aber der Herr Ungarn an und gab ihm einen Schützer, der in der Menge seiner Stärke dahergeht. Der Herr gab nämlich die Herrschaft (das Königtum) Géza, seinem Diener. Er wurde am Tag der Heiligen Cecilia gekrönt. ${ }^{13}$ Dessen rechte Hand ergriff und verstärkte er, und er unterwarf ihm viele Völker und vor dem Gesicht seines Schwertes wandte er die Rücken der Könige zur Flucht.

Zur ersten Periode gibt die Textausgabe von Domanovszky keinen locus an (auch die späteren Kommentare und Übersetzungen nicht): ,, Respexit autem Dominus Hungariam ... “ Die Bedeutung von respicio, 'jemanden mit Gnade ansehen', ist im christlichen Latein ausdrücklich ein Hebraismus. Es kommt oft vor, so in Ex 2,25: „et respexit Dominus filios Israel, et cognovit eos". (Dies passiert, als die Lage der Abkömmlinge Jakobs sich in Ägypten verschlimmert hat.) Eine Textausgabe hat natürlich nicht die Aufgabe, einen Hebraismus, der oft vorkommt, aufzuzeigen - das ist erst die Aufgabe eines sprachlichen Kommentars, wenn der biblische sprachliche Hintergrund (Kontext) für den Leser nicht eindeutig ist. (Vieles deutet darauf hin, dass gegeben

${ }^{12}$ Abweichend von der Edition wird der lateinische Text mit normalisierter Orthographie wieder-

${ }^{13}$ Im Jahre 1141, am 22. Februar. 
dieser auch bei der Ausgabe von 1937 nicht eindeutig war ...) Das Wort respexit ist jedoch für einen mittelalterlichen, aber auch für einen heutigen Menschen, der das Stundengebet absolviert, spezifisch bekannt aus dem Magnificat. Auch das müsste erst ein Kommentar registrieren. Das Respexit Dominus humilitatem ist aber an vielen Orten die Magnificat-Antiphon des Mittwochs in der Zeit „per annum“. ${ }^{14}$ Deshalb ist der Satz des Gesanges als Parallele anzugeben.

„... et dedit propugnatorem gradientem in multitudine fortitudinis suae. “ Dass die Philologen und Übersetzer den biblischen Ursprung nicht recherchiert haben, ist an dieser Stelle schon peinlich, denn die biblische Formel springt ins Auge. Schon bei dedit hätte man auf das Magnificat aufmerksam werden müssen. (So formuliert man im nicht-biblischen Latein nicht - man kann allerdings nicht eine konkrete Stelle angeben, weil Gott so viel und so viele Dinge gegeben hat, dedit.) Aber die Fortsetzung ist so eigenartig, dass sie auch in der einfachsten Konkordanz aufzufinden ist, wenn der Philologe sich nicht daran erinnerte. Der mittelalterliche Mensch hat sich aber mit Sicherheit erinnert: So fängt die erste alttestamentliche Lesung in der Messe des Mittwochs in der Karwoche, ein interessanter Text vom Propheten Jesaja, an:

Lectio Isaiae prophetae (Is 62,11; 63,1-7) Haec dicit Dominus Deus. Dicite filiae Sion : ecce, Salvator tuus venit : et merces eius cum eo : et opus illius coram illo. Quis est iste, qui venit de Edom : tinctis vestibus de Bosra? Iste formosus in stola sua gradiens : in multitudine fortitudinis suae. Ego, qui loquor iustitiam : et propugnator sum, ad salvandum.

Nach meinen heutigen Kenntnissen hat wahrscheinlich der Chronist selbst den biblischen Text paraphrasiert. Wenn er damit gerechnet hat, dass seine Leser den biblischen Ursprung wiedererkennen, dann kann man als Ertrag des Textes für die Darstellung der Königsherrschaft nennen, dass die körperliche Schönheit (formosus), die Stola (stola), das Reden der Wahrheit (loquor iustitiam) und die Rettung (ad salvandum) als auf den König bezogene Momente aufgefasst werden können. In einer wirklich informierenden Ausgabe müsste der Ursprung des Hinweises angegeben werden: cf. Is 63,1 (lc Qu/h6/f4) (Lesung in der Messe des Mittwochs der sechsten Fastenwoche, d.h. der Karwoche). Der Text ist eine Paraphrase von Is 63,1, aber das Bild wird dadurch nuanciert, dass das Wort propugnator in der lateinischen Bibel selten ist. Das zweite Vorkommen findet sich ebenfalls beim Propheten Jesaja:

Clamabunt Aegyptii ad Dominum a facie tribulantis : et mittet eis Salvatorem : et propugnatorem : qui liberet eos. Et cognoscetur Dominus ab Aegypto : et cognoscent Aegyptii Dominum in die illa. (Is 19,20-22)

Dieser Text ist die erste alttestamentliche Lesung in der Messe des Samstags der Quatember im Advent (lc Adv/4t/sb). Merkenswert sind die liturgische Zeit und der litur-

\footnotetext{
${ }^{14}$ Respexit Dominus humilitatem meam et fecit in me magna quia potens est (CAO-4620). In Gran (d.h. im Graner Ritus in Ungarn) wurde diese Antiphon nicht benutzt, andernfalls war sie fast überall (z.B. bei den Mönchsorden) verbreitet. Deshalb können wir sie als bekannt voraussetzen.
} 
gische Ort. (Und auch, dass das respexit Dominus, wenn auch in einem anderen Kontext, auch in Bezug auf die Ägypter geschrieben ist, wie wir gesehen haben.)

„Dedit enim Dominus regnum Geythae, puero suo. “ Die Wendung dedit regnum ist vielleicht nicht ein Zitat, das man an einen biblischen locus binden kann, sondern vielmehr ein biblisches sprachliches Element, ein Hebraismus. Das Muster der Wendung puero suo ist, wie es auf der Hand liegt, die Phrase suscepit Israel puerum suum (L 1,54) aus dem Magnificat (cf. respexit!).

Man muss zum folgenden kurzen Satz keinen biblisch-liturgischen Hintergrund suchen: , Qui in die Sanctae Caeciliae virginis coronatus est"

Umso eindeutiger ist aber der letzte Satz des Kapitels: „, Cuius dexteram apprehendit et confortavit ... " Dieser Text ist eine Paraphrase des Folgenden:

Lectio Isaiae prophetae (Is 45,1-8) Christo meo Cyro : cuius apprehendi dexteram : ut subiciam ante faciem eius gentes : et dorsa regum convertam.

Es ist ein Rätsel, warum der Textherausgeber der Chronica, Sándor Domanovszky, in den Notizen den Text nur von subiciam an zitiert, warum er ihn nur von dort an für eine Parallele hält. Dieser Teil wird nämlich auch nur paraphrasiert zitiert: ,,... subiecitque ei gentes multas 'et dorsa regum vertit' in fugam 'a facie gladii' eius. “"

Des Weiteren, warum hält er das Wort vertit für ein genaueres Zitat (die Zeichen , , weisen auf genaue Zitate hin), und wenn er schon die Wendung a facie gladii für ein Zitat hält, warum zitiert er dann nicht zu gentes multas den Psalmtext (Ps 134,10), der sich im liturgischen Usus leicht fixieren lässt? (Übrigens ist die Wendung wiederum nur ein Hebraismus, den man nicht an eine konkrete Bibelstelle binden kann.)

Es ist wichtig, dass wir philologisch bestimmen, dass dieser Text ein Zitat ist; es ist aber noch bedeutender, dass der berühmte Messias-Text eine alttestamentliche Lesung des Samstags der Quatember im Advent ist (lc Adv/4t/sb), bei der es sich, ähnlich wie bei Is 63,1, ausdrücklich um eine Prophetie über den Erlöser, Salvator, handelt (Is 45,8).

Die Identifizierung der beiden Ausschnitte aus den Prophetien von Jesaja, die Feststellung, dass sie als Hypotexte, als Grundlage der Paraphrase dienten, lässt sich nicht widerlegen. Beide Texte sind nicht nur durch ihre Bilder und spezifischen Wendungen auffallend, sondern lassen sich an solche, und zwar nur an solche liturgische Zeiten binden, die sich im liturgischen Jahr besonders hervorheben. Der Samstag der Quatember im Advent und der erste Sonntag in der Fastenzeit sind ohne Zweifel solche Zeiten und solche liturgischen Orte.

Man könnte auch die verborgeneren Fäden beobachten: v.a. das Motiv des Salvators, und dass auch im anderen alttestamentlichen Text des letzteren Zeitpunktes im liturgischen Jahr (s. oben) das seltene Wort propugnator zu finden ist (zusammen mit Salvator). Die Assoziationsfähigkeit des mittelalterlichen Menschen war gewiss feiner als die unsere. Man kann auch erahnen, dass sie die charakteristische Wendung des einleitenden Satzes v.a. aus einer bekannten Antiphon am einfachsten identifizieren konnten. 
Der kurze Text lässt sich, was die Technik seiner Herstellung betrifft, so charakterisieren: Er konstituiert sich durch Zusammenfügen von durch liturgische Verwendung hervorgehobenen biblischen „Versen“ in der Form der Paraphrasierung, d.h. Cento, wenn man will: Collage.

Wir sollten den Benutzer der Textausgabe vor der Mühe der Erforschung der liturgischen Verwendung bzw. vor dem Versagen ihrer Erforschung schonen. Wenn wir das alles nicht in Betracht ziehen, wenn wir die mehr als wahrscheinliche liturgische Vermittlung nicht aufzeigen, wenn wir also nur den biblischen Text als letzte Quelle angeben (und sogar diese unvollständig), dann haben wir dem Leser, der unserer Textausgabe vertraut, nicht nur nicht geholfen, sondern ihn geradezu irregeführt.

Die Mediävistik muss die mittelalterliche liturgische Kultur erlebbar, spürbar, erfahrbar machen: diese große, im Grunde genommen homogene Kultur, in der die Herstellung, Rezeption und Deutung neuerer Texte auf einem im Gedächtnis gespeicherten, gemeinsamen, gesprochen-gesungenen Textkorpus basierte.

\author{
Balázs Déri Univ.-Prof. \\ Seminar für Latinistik \\ Eötvös-Loránd-Universität (ELTE) Budapest \\ Ungarn \\ deri.balazs@btk.elte.hu
}

